\title{
Robustness margin need not be a continuous function of the problem data
}

\section{B.R. Barmish}

Department of Electrical and Computer Engineering, University of Wisconsin-Madison, Madison, WI 53706, U.S A

\section{P.P. Khargonekar}

Department of Electrical Engineering and Computer Science Unwersity of Michigan, Ann Arbor, MI 48109, US A

\section{Z.C. Shi}

Department of Electrical and Computer Engineerıng. University of Wisconsin-Madison, Madison, WI 53706, U S.A

\section{R. Tempo \\ CENS-CNR, Polltecnico di Torino, Corso Duca degh Abruzzı 24, 10129 Torıno, Italy}

Received 20 January 1990

Revised 13 Apnl 1990

Abstract. For systems with structured real perturbations, it is shown that the robustness margin for stability can be a discontınuous function of the problem data

Keywords Robustness, stability; uncertainty; ill conditıoning.

\section{Introduction}

Consıder a linear control system with a transfer function or state space description parameterized in terms of a vector of uncertain parameters $q \in \mathbf{R}^{\prime}$. A fundamental problem addressed in a large number of papers is: Determune the maximum uncertainty bound, call it $r_{\max }$, such that the system is stable for all $q \in \mathbf{R}^{\ell}$ with $\|q\| \leq r_{\max }$. Note that the chosen norm for $q$ is almost always $\ell^{2}$ or $\ell^{\infty}$ and $r_{\max }$ is generally called the robustness margin; see Section 2 for a precise definition.

In many cases, a slightly different formulation of the problem above is considered; 1.e., given an uncertainty bound $r>0$, determine if the system is stable for all $q \in \mathbf{R}^{\ell}$ with $\|q\| \leq r$. In this case, only a 'yes' or 'no' answer is required. In the sequel, all analysis is carried out in the robustness margin framework but it should be noted that the consequences apply equally well to this alternative yes/no formulation; e.g., see the discussion associated with the example in Section 3.

The simple paradigms above are at the heart of many robust stability analysıs technıques; e.g., see the literature ranging from real $\mu$ as in Doyle [1] and Packard [2] to the post-Kharitonov [3] literature (see Barmish [4] and Jury [5] for reviews of the continuous-time and discrete-tıme cases respectively), to polytope stability problems as in Bartlett, Hollot and Huang [6] and to the theory dealing with frequency sweeping methods; e.g., see de Gaston and Safonov [7], Biernack,, Hwang and Bhattacharryya [8], Hinrichsen and Pritchard [9] and Barmish [10].

Our main technical objective in this paper is to demonstrate that the robustness margin $r_{\max }$ is not necessarty continuous with respect to the problem data; the notion of problem data will be fully explained in the sequel. This discontınuity phenomenon is seen to be independent of the computational algorithm used to actually calculate $r_{\max }$. Matters are further complicated by the fact that at the point of discontınuity in the space of problem data, the robustness margin may be much smaller than at neighboring points. This may lead to potentially deceptive conclusions.

We feel that the most important implication of the present paper is that there is a serious issue pertaining to conditioning properties of the robustness margin Despite the possibility that discontinuity of this margin may be nongeneric, in regions close to the discontinuity set, ill conditioning of $r_{\max }$ must nevertheless be addressed. Therefore, our conclusion is that a thorough analysis of conditioning properties of the robust stability problem is an important area for future research.

The case which we make for discontunuity of the robustness margin is based on a simple example of a unity feedback system - the plant has 
uncertain parameters entering linearly into numerator and denominator coefficients. We call this a linear uncertainty structure. Using $d$ to represent the data describing the system, the robustness margin is written explicitly as $r_{\max }(d)$ and we prove the following: There exusts a sequence of data $\langle d(n)\rangle_{n=1}^{\infty}$ converging to some $d^{*}$ such that

$\lim _{n \rightarrow \infty} r_{\max }(d(n))>r_{\text {max }}\left(d^{*}\right)$

That is, if one solves the sequence of robustness margins problems corresponding to $d(n)$, the margins $r_{\text {max }}(d(n))$ may differ considerably from $r_{\max }\left(d^{*}\right)$ This happens even as the data $d(n)$ gets arbitranly close to $d^{*}$.

For the simple case of linear uncertainty structures as in Section 3, it is felt that it should be possible to perform some sort of apnon check for discontinuity However, the fact that the discontinuity phenomenon occurs at the level of linear uncertainty structures serves as a 'warning' that care must be exercised when dealing with more complicated nonlinear problems. This is consistent with the example involving a nonlinear uncertainty structure in the paper by Ackermann, Hu and Kaesbauer [11] - severe computational problems arise anse as a certain data parameter is changed

\section{Notation and definition of the robustness margin}

We consider polynomials with real coefficients $a_{r}$ which depend continuously on a vector of uncertain parameters $q \in \mathbf{R}^{\ell}$ whose $t$-th component is $q$, To denote the dependence of $a$, on $q$, we write $a_{1}(q)$. Hence, we take an uncertain polynomial to be of the form

$p(s, q)=s^{m}+\sum_{i=0}^{m-1} a_{i}(q) s^{\prime}$.

In Section 3, $a_{1}(q)$ is affine linear and in Section 4, $a_{i}(q)$ is multilinear. When $q=0$, we obtain the so-called nominal polynomial $p(s, 0)$, which is assumed to be strictly stable; i.e., its roots lie in the open left half plane.

A bounding set for the vector of uncertain parameters $q$ will be a box parametenzed by its radius $r$; this box 1 denoted as $Q_{r}$ and is described by

$Q_{r} \doteq\left\{q \in \mathbf{R}^{l}:\left\|q_{i}\right\| \leq r ; l=1,2, . ., l\right\}$.
Note that the discussion to follow can easily be adapted to handle the case when $Q_{r}$ is a sphere; 1 e., the discontinuity phenomenon is not particular to the $\ell_{\infty}$ norm on uncertain parameters. In addition, discontınuities can occur when workıng with many other stability regions besides the open left half plane - the unit disk being a prime example.

Robustness margin In accordance with the discussion in Section 1, the robustness margin (for stability) is given by

$r_{\mathrm{m} 2 \mathrm{x}} \doteq \sup \{r: p(s, q)$ is strictly stable
for all $\left.q \in Q_{r}\right\}$.

Dependence on problem data. In each of the examples to follow, the integers

$l=\operatorname{dim} q$ and $m=\operatorname{deg} p(s, q)$

are held fuxed and problem data consists of the coefficient functions $a_{0}(), a_{1}(), \ldots, a_{m-1}()$ To illustrate the discontinuity phenomenon, we use a finite-dimensional space for this problem data. That is, each $a_{1}(\cdot)$ is viewed as a mapping on data vectors $d \in \mathbf{R}^{p}$ to continuous functions of $q$ For example, a family of problems might be described by $p=6, l=2, m=2$ and

$$
\begin{gathered}
p(s, q)=s^{2}+\left(d_{1}+d_{2} q_{1}+d_{3} q_{2}\right) s \\
+\left(d_{4}+d_{5} q_{1}+d_{6} q_{2}\right)
\end{gathered}
$$

A spectfic robustness margin problem is obtained with $d_{1}=2, d_{2}=1, d_{3}=4, d_{4}=3, d_{5}=6$ and $d_{6}=12$. This leads to

$$
p(s, q)=s^{2}+\left(2+q_{1}+4 q_{2}\right) s+\left(3+6 q_{1}+12 q_{2}\right) \text {. }
$$

Within this data space framework, two problems are deemed to be 'close together' if their assoclated data vectors (call them $d^{1}$ and $d^{2}$ ) are close together in some arbitrary but fixed norm on $\mathbf{R}^{p}$; i.e., $\left\|d^{1}-d^{2}\right\|$ is small.

To denote dependence on $d$, we henceforth write $p_{d}(s, q)$ and $r_{\text {max }}(d)$ in lieu of $p(s, q)$ and $r_{\max }$ respectively We are now prepared to present our main example.

\section{Example establishing discontinuity of the robust- ness margin}

Before formally proceeding, it is important to note that it is easy to construct relatively trivial 
examples for which discontinuity of $r_{\max }$ can easily be demonstrated. Such examples involve cases when there is only one uncertain parameter, cases when the uncertainty structure is highly nonlinear, cases when the limiting polynomial $p_{d^{*}}(s, q)$ is only marginally stable and cases when $p_{d^{*}}(s, q)$ is structurally different from $p_{d(n)}(s, q)$, e.g, $p_{d^{*}}(s, q)$ has lower degree or a smaller number of uncertainties than $p_{d^{*}}(s, q)$. In contrast, the example below is simple yet nontrivial.

Indeed, consider a unity feedback system with open loop transfer function denoted by

$P_{d}(s, q)=K_{d} \frac{N_{d}(s, q)}{D_{d}(s, q)}$

where $N_{d}(s, q)$ and $D_{d}(s, q)$ are uncertain polynomials and $K_{d}$ is the loop gain. The subscript ' $d$ ' is used to emphasize dependence on the data. In this example, $l=2, m=4$ and with $d=d^{*}$, consider

$K_{d^{*}}=a$,

$N_{d^{*}}(s, q)=4 a+10 a q_{1}$,

and

$$
\begin{aligned}
D_{d^{*}}(s, q)= & s^{4}+\left(20-20 q_{2}\right) s^{3} \\
& +\left(44+2 a+10 q_{1}-40 q_{2}\right) s^{2} \\
& +\left(20+8 a+20 a q_{1}-20 q_{2}\right) s+a^{2},
\end{aligned}
$$

where

$a \doteq 3+2 \sqrt{2}$.

Using our data notation, we write

$K_{d}=d_{0}$,

$N_{d}(s, q)=d_{1}+d_{2} q_{1}$,

and

$$
\begin{aligned}
D_{d}(s, q)= & s^{4}+\left(d_{3}+d_{4} q_{2}\right) s^{3} \\
& +\left(d_{5}+d_{6} q_{1}+d_{7} q_{2}\right) s^{2} \\
& +\left(d_{8}+d_{9} q_{1}+d_{10} q_{2}\right) s+d_{11} .
\end{aligned}
$$

By comparing the expressions for $K_{d^{*}}, N_{d^{*}}(s, q)$ and $D_{d}(s, q)$ with $K_{d}, N_{d}(s, q)$ and $D_{d}(s, q)$, respectively, it is clear that the $d_{1}^{*}$ are readily available, e.g., $d_{0}^{*}=a, d_{1}^{*}=4 a, d_{2}^{*}=10 a, d_{3}^{*}$ $=20$, etc.
Now, we consider the data sequence $\langle d(n)\rangle_{n=1}^{\infty}$ described by

$d_{i}(n)= \begin{cases}d_{t}^{*} & \text { for } i \neq 0, \\ a_{n} & \text { for } t=0,\end{cases}$

where

$a_{n} \doteq a-1 / n$.

This sequence corresponds to the case where the plant data is fixed and the gain $a_{n}$ is converging to $a$.

Robustness margin. In order to obtain the robustness margin along the $d(n)$ sequence for the feedback system above, we study the closed loop polynomial

$$
\begin{aligned}
p_{d(n)}(s, q)= & K_{d(n)} N_{d(n)}(s, q)+D_{d(n)}(s, q) \\
= & s^{4}+\left(20-20 q_{2}\right) s^{3} \\
& +\left(44+2 a+10 q_{1}-40 q_{2}\right) s^{2} \\
& +\left(20+8 a+20 a q_{1}-20 q_{2}\right) s \\
& +a\left(5 a-\frac{4}{n}+10\left(a-\frac{1}{n}\right) q_{1}\right)
\end{aligned}
$$

and for the limiting case, we study the closed loop polynomal

$$
\begin{aligned}
p_{d^{*}}(s, q)= & a N_{d^{*}}(s, q)+D_{d^{*}}(s, q) \\
= & s^{4}+\left(20-20 q_{2}\right) s^{3} \\
& +\left(44+2 a+10 q_{1}-40 q_{2}\right) s^{2} \\
& +\left(20+8 a+20 a q_{1}-20 q_{2}\right) s \\
& +\left(5 a^{2}+10 a^{2} q_{1}\right) .
\end{aligned}
$$

Discontinuity claim (see next subsection for proof). We claim that

$0.417 \approx \lim _{n \rightarrow \infty} r_{\max }(d(n))>r_{\max }\left(d^{*}\right)=0.234$.

That $1 \mathrm{~s}$, we claim that the robustness margin is discontinuous at the data point $d^{*}$. In fact, for this example, along the data sequence $\langle d(n)\rangle_{n=1}^{\infty}$, the robustness margin is given by

$\lim _{n \rightarrow \infty} r_{\max }(d(n))=1-\frac{1}{10} a \approx 0.417$ 
However, precisely at $d^{*}$, the robustness margin becomes

$r_{\max }\left(d^{*}\right)=\frac{7-a}{5} \approx 0234$.

This example illustrates the 'false sense of secur1ty' associated with the robustness margin. To further elaborate, if $q_{1}^{*}=q_{2}^{*} \approx 0234$, two of the roots of the closed loop polynomial $p_{d(n)}\left(s, q^{*}\right)$ approach the imaginary axis as $n \rightarrow \infty$. That is, $p_{d(n)}\left(s, q^{*}\right)$ is 'nearly' destabilized by an uncertainty vector $q^{*}$ whose norm is 0.234 despite the fact that the predicted margin is approximately 0417

Proof of claim. Along the data sequence, we examine the closed loop polynomial $p_{d(n)}(s, q)$ given by (1). Then, to obtain the robustness margin, we use the fact that the leading minors of the Hurwitz testing matrix must be positive. This leads to the following four conditions:

Condition 1:

$\left(20-20 q_{2}\right)>0$

Condition 2.

$$
\begin{gathered}
\left(20-20 q_{2}\right)\left(44+2 a+10 q_{1}-40 q_{2}\right) \\
-\left(20+8 a+20 a q_{1}-20 q_{2}\right)>0 .
\end{gathered}
$$

\section{Condition 3}

$$
\begin{aligned}
& \left(20-20 q_{2}\right)\left(44+2 a+10 q_{1}-40 q_{2}\right) \\
& \left(20+8 a+20 a q_{1}-20 q_{2}\right) \\
& -\left(20-20 q_{2}\right)^{2}\left(5 a^{2}-\frac{4}{n} a+10 a\left(a-\frac{1}{n}\right) q_{1}\right) \\
& -\left(20+8 a+20 a q_{1}-20 q_{2}\right)^{2}>0 .
\end{aligned}
$$

\section{Condition 4:}

$5 a^{2}-\frac{4}{n} a+10 a\left(a-\frac{1}{n}\right) q_{1}>0$.

Note that $r_{\max }(d(n))$ is the supremal value of $r$ such that the four inequalities above hold for all $q \in Q$, That is, letting

$r_{t}(d(n))=\sup (r$. Condition $\imath$ holds

$$
\text { for all } \left.q \in Q_{r}\right\} \text {, }
$$

It follows that

$$
r_{\max }(d(n))=\min _{t=1, \ldots 4}\left\{r_{i}(d(n))\right\} \text {. }
$$

The remainder of the proof will proceed via a number of steps

Step 1. We claim that

$\lim _{n \rightarrow \infty} r_{3}(d(n))=1-\frac{a}{10} \approx 0.417$

To prove this claim, it is first verified that Condition 3 is equivalent to

$$
\begin{aligned}
& 2\left[4 \sqrt{a}-20+10 \sqrt{a} q_{1}+20 q_{2}\right]^{2} \\
& \cdot\left(20-2 a-20 q_{2}\right) \\
& \quad+\frac{1}{n} a\left(4+10 q_{1}\right)\left(20-20 q_{2}\right)^{2}>0 .
\end{aligned}
$$

Now, to obtain the quantity $\lim _{n \rightarrow \infty} r_{3}(d(n))$, we rewrite the inequality above as

$2 F_{1}(q) F_{2}(q)+G_{n}(q)>0$

where

$F_{1}(q)=\left[4 \sqrt{a}-20+10 \sqrt{a} q_{1}+20 q_{2}\right]^{2}$,

$F_{2}(q)=\left(20-2 a-20 q_{2}\right)$,

and

$G_{n}(q)=\frac{1}{n} a\left(4+10 q_{1}\right)\left(20-20 q_{2}\right)^{2}$

To establish the desired limit for $r_{3}(d(n))$, we observe that if $q_{2}=1-\frac{1}{10} a$ and $q_{1}=-04$, then

$2 F_{1}(q) F_{2}(q)+G_{n}(q)=0$.

That is, for all $n$,

$r_{3}(d(n)) \leq 1-\frac{1}{10} a$

Furthermore, it is also easy to venfy that for arbitrarily small $\varepsilon>0$, there exists an integer $N_{\varepsilon}$ having the following property: For any fixed $n>$ $N_{\varepsilon}$ and any uncertainty $q \in Q_{1-a / 10-\varepsilon}$,

$2 F_{1}(q) F_{2}(q)+G_{n}(q)>0$.

Hence, for $n>N_{e}$,

$r_{3}(d(n)) \geq 1-\frac{1}{10} a-\varepsilon$

From the two inequalities involving $r_{3}(d(n))$ above, we conclude that

$\lim _{n \rightarrow \infty} r_{3}(d(n))=1-\frac{1}{10} a$.

Hence, the claim is established 
Step 2: We claim that for $n$ sufficiently large,

$r_{1}(d(n))>r_{3}(d(n))$

for $t=1,2$, 4. Indeed from Condition 1 , it is trivial to see that

$r_{1}(d(n))=1$.

To verify (3) for $r_{2}(d(n))$, we new the left hand side of Condition 2 as a function of $\left(q_{1}, q_{2}\right)$ It suffices to show that this function is positive on a box of radius $r=1-\frac{1}{10} a$. To this end, notice that for arbitrary $\left|q_{1}\right| \leq r<0.5$ and $q_{2} \leq r<0.5$, it is easy to verify that we have crude bounds

$\left(20-20 q_{2}\right)\left(44+2 a+10 q_{1}-40 q_{2}\right)>300$

and

$20+8 a+20 a q_{1}-20 q_{2}<150$.

Therefore, the left hand side in Condition 2 remains positive as required.

Finally, setting the left hand side of Condition 4 to zero, it is straightforward to obtain the formula

$r_{4}(d(n))=\frac{5 n a-4}{10(n a-1)}$.

Hence, it is easy to see that

$\lim _{n \rightarrow \infty} r_{4}(d(n))=\frac{1}{2}$

which umplies that $r_{4}(d(n))>r_{3}(d(n))$ for $n$ sufficiently large.

Step 3: We claim that

$\lim _{n \rightarrow \infty} r_{\max }(d(n))=1-\frac{1}{10} a \approx 0.417$.

This claim follows easily from Steps 1 and 2 . That is, we have

$$
\begin{aligned}
\lim _{n \rightarrow \infty} r_{\max }(d(n)) & =\lim _{n \rightarrow \infty} r_{3}(d(n)) \\
& =1-\frac{1}{10} a \approx 0.417 .
\end{aligned}
$$

Step 4: We claim that

$r_{\max }\left(d^{*}\right)=\frac{7-a}{5} \approx 0.234$.

Indeed, as in the $d(n)$ analysis, we use the formula

$r_{\max }\left(d^{*}\right)=\min _{i=1, \ldots, 4}\left\{r_{i}\left(d^{*}\right)\right\}$ where $r_{t}\left(d^{*}\right)$ is obtained from the $i$-th Hurwitz inequality at $d^{*}$. Analogous to Steps $1-3$, we first analyze Condition 3 with $n \rightarrow \infty$. By a straightforward computation, it is easy to verify that Condition 3 is equivalent to

$$
\begin{gathered}
2\left(4 \sqrt{a}-20+10 \sqrt{a} q_{1}+20 q_{2}\right)^{2} \\
\cdot\left(20-2 a-20 q_{2}\right)>0 .
\end{gathered}
$$

Now, we examine each factor separately and obtain the margin

$r_{3}\left(d^{*}\right)=\frac{7-a}{5} \approx 0.234$.

Next, reasoning exactly as in Steps $2-3$, it is easy to venfy that

$$
\begin{aligned}
& r_{1}\left(d^{*}\right)=1>r_{3}\left(d^{*}\right), \\
& r_{2}\left(d^{*}\right)>r_{3}\left(d^{*}\right), \\
& r_{4}\left(d^{*}\right)=\frac{1}{2}>r_{3}\left(d^{*}\right) .
\end{aligned}
$$

Hence, from (4), we obtain

$r_{\max }\left(d^{*}\right)=r_{3}\left(d^{*}\right)=\frac{7-a}{5} \approx 0.234$.

The proof of the claim is now complete.

Yes-No Problem. The discontinuity claim above can also be interpreted in terms of the yes/no problem formulation discussed in the Introduction. To illustrate, consider a robust stability problem with given uncertainty bound $r=0.3$. Now, the following problem arises: When using $d^{*}$, the answer to the robust stability question is "no" but taking $d(n)$ the answer is "yes".

Remarks. In practice, the robustness margin can be computed va a number of methods. For example, instead of using a Hurwitz matrix as in the proof of the claim above, one can use the wellknown frequency sweep method. That is, letting

$$
\begin{array}{r}
r_{\max }(d, \omega) \doteq \sup \left\{r: p_{d}(j \omega, q) \neq 0\right. \\
\text { for all } \left.q \in Q_{r}\right\},
\end{array}
$$

it then follows that

$$
r_{\max }(d)=\inf _{\omega} r_{\max }(d, \omega)
$$


In our specific example, solution by frequency sweep method for $n$ finite leads to

$$
\begin{aligned}
& r_{\max }(d(n), \omega) \\
& \quad= \begin{cases}\max \left\{C_{n}(\omega), D_{n}(\omega)\right\} & \text { if } \omega \neq 0, \\
\frac{5 n a-4}{10(n a-1)} & \text { if } \omega=0,\end{cases}
\end{aligned}
$$

where

$$
\begin{aligned}
& C_{n}(\omega)=\left|\frac{\left(\omega^{2}-a\right)^{2}\left(\omega^{2}-1\right)}{10 \Delta_{n}(\omega)}-04\right|, \\
& D_{n}(\omega)=\left|\frac{a\left(\omega^{2}-a\right)^{2}}{10 \Delta_{n}(\omega)}-1\right|,
\end{aligned}
$$

and

$$
\Delta_{n}(\omega)=\omega^{4}-a\left(2-\frac{1}{n}\right) \omega^{2}+a\left(a-\frac{1}{n}\right) .
$$

In the limiting case, we obtain

$$
r_{\max }\left(d^{*}, \omega\right)= \begin{cases}\max \left\{\left|\frac{\omega^{2}-5}{10}\right|,\left|1-\frac{a}{10}\right|\right\} \\ \frac{1}{\frac{1}{2}} & \text { if } \omega \neq 0, \omega \neq \sqrt{a}, \\ \frac{1}{5}(7-a) & \text { if } \omega=\sqrt{a}\end{cases}
$$

\section{More complicated uncertainty structures}

Given the uncertain polynomial $p_{d}(s, q)$, it is natural to wonder whether some simple apriori test for discontınuity of $r_{\max }(d)$ is possible Although we feel that this may ultimately prove to be the case at the basic level of lnear uncertainty structures, the apnon detection of discontinuity may be extremely diffıcult for more complicated uncertainty structures.

In this section, we show that examples of discontinuity for multılunear uncertainty structures are easily created by 'embedding' the polynomal given in Section 3 into a more complicated structure in such a way that it may not be apparent what is causing the trouble. To this end, let

$g_{d(n)}(s, q)=p_{d(n)}(s, q) f(s, q)$

where $P_{d(n)}(s, q)$ is given by (1) and

$f(s, q)=s^{2}+\left(1+q_{2}\right) s+\left(1+q_{3}\right)$.
Similarly, we take

$g_{d^{*}}(s, q)=p_{d^{*}}(s, q) f(s, q)$

where $p_{d^{*}}(s, q)$ is given by (2). Note that the uncertainty structures above are multilinear and the first factors in $g_{d(n)}(s, q)$ and $g_{d^{*}}(s, q)$ are the same polynomials which were used in Section 3. Moreover, since the robustness margin of $f(s, q)$ is unity, it follows that the margins for $g_{d(n)}(s, q)$ and $g_{d^{*}}(s, q)$ are exactly the same as those found for $p_{d(n)}(s, q)$ and $p_{d^{*}}(s, q)$ in Section 3 Notice that if $g_{d(n)}(s, q)$ and $g_{d^{*}}(s, q)$ are given in expanded form rather than factored as above, the detectıon of the discontınuity becomes much more difficult. That is, if one has a theory to flag discontinuity at the level of affine linear uncertainties, then one is faced with a complicated factorization problem. As seen below, matters can be even worse because it is easy to construct examples for which a factorization does not exits

Modification which does not permit factorization. Take $f(s, q), g_{d(n)}(s, q), g_{d^{*}}(s, q), p_{d(n)}(s, q)$, $p_{d^{*}}(s, q), d(n)$ and $d^{*}$ as above and let $\left\langle b_{n}\right\rangle_{n=1}^{\infty}$ be any sequence of positive real numbers converging to zero. Now, using the polynomials $g_{d(n)}(s, q)+b_{n}$ and $g_{d^{*}}(s, q)$, it can be shown (see Appendix) that the same discontinuity phenomenon occurs along appropriately constructed subsequences $\left\langle d\left(n_{j}\right)\right\rangle_{J=1}^{\infty}$ of $\langle d(n)\rangle_{n=1}^{\infty}$ and $\left\langle b_{n_{k}}\right\rangle_{k=1}^{\infty}$ of $\left\langle b_{n}\right\rangle_{n=1}^{\infty}$. Moreover, by examination of the coefficients of $s^{0}$, it is easy to see that a factorization of the modified polynomials into a product of polynomials each having affine uncertainty structure is impossible. That is, it is straightforward to verify that the quantity

$$
\begin{aligned}
\operatorname{coef}\left(s^{0}\right)= & a\left(5 a-\frac{4}{n}+10\left(a-\frac{1}{n}\right) q_{1}\right)\left(1+q_{3}\right) \\
& +b_{n}
\end{aligned}
$$

cannot be factored as a product of two affine linear functions of $q$.

\section{Conclusion}

In view of the arguments and examples given in this paper, it is felt that investigation into conditioning properties of the robust stability problem is an important area for future research. 


\section{Appendix}

We consider the same setup as in Section 3; 1.e., $p_{d}(s, q)$ is a monic polynomial with robustness margin $r_{\max }(d)$ and $\langle d(n)\rangle_{n=1}^{\infty}$ is a sequence converging to $d^{*}$. Given any $\varepsilon>0$, define

$\tilde{p}_{d}(s, q, \varepsilon)=p_{d}(s, q)+\varepsilon$

whose robustness margin is denoted by $\tilde{r}_{\max }(d, \varepsilon)$. Now, we establish a basic lemma.

Lemma. Suppose that for each $n$,

$r_{\max }(d(n))>\beta$.

Then there exists an integer $N$ and a sequence $\left\langle\varepsilon_{n}\right\rangle_{n=1}^{\infty}$ of positive numbers converging to zero such that

$\tilde{r}_{\max }\left(d(n), \varepsilon_{n}\right)>\beta$

for all $n \geq N$.

Proof. Since $d(n)$ converges to $d^{*}$, pick $N$ such that

$\left|d(n)-d^{*}\right| \leq 1$

for all $n \geq N$. Now, letting $n \geq N$ be fixed, the proof continues with a sequence of claims.

Claim 1. There exists an $\omega_{0}>0$ having the following property: For arbitrary $|\omega|>\omega_{0}, q \in$ $Q_{\beta}$ and $\varepsilon \in[0,1]$,

$\left|\tilde{p}_{d(n)}(\jmath \omega, q, \varepsilon)\right| \neq 0$.

This claim is easily established after noting that for $\omega$ sufficiently large,

$$
\begin{aligned}
& \omega^{m}>\max \left\{\left|\tilde{p}_{d}(\jmath \omega, q)-(\jmath \omega)^{m}\right|:\right. \\
& \left.\qquad q \in Q_{\beta} ; \varepsilon \in[0,1] ;\left|d-d^{*}\right| \leq 1\right\} .
\end{aligned}
$$

Claim 2. With $\omega_{0}$ as in Claim 1 and $\varepsilon \in[0,1]$, let

$F_{n}(\varepsilon) \doteq \min \left\{\left|\tilde{p}_{d(n)}(\mathrm{j} \omega, q, \varepsilon)\right|: q \in Q_{\beta} ;|\omega| \leq \omega_{0}\right\}$.

Then

$F_{n}(0)>0$.

This claim is establıshed by contradiction. Indeed, if $F_{n}(0)=0$, it follows that $\left|\tilde{p}_{d(n)}\left(j \omega^{*}, q^{*}, 0\right)\right|=0$ for some $\left|\omega^{*}\right| \leq \omega_{0}$ and $q^{*} \in Q_{\beta}$. Hence,

$r_{\max }(d(n))=\tilde{r}_{\max }(d(n), 0) \leq \beta$,

which is a contradiction.
Claim 3. $F_{n}(\varepsilon)$ is continuous with respect to $\varepsilon \in[0,1]$. This claim follows from continuous dependence of $\tilde{p}_{d(n)}(j \omega, q, \varepsilon)$ on $(\omega, q, \varepsilon)$ and compactness of $\left[-\omega_{0}, \omega_{0}\right]$ and $Q_{\theta}$. That is, $F_{n}(\varepsilon)$ is an infumal value function; e.g., see Berge [12]

Claim 4. There exists some $\varepsilon_{n}^{*}>0$ such that

$F_{n}(\varepsilon)>0$

for all $\varepsilon \in\left[0, \varepsilon^{*}\right]$. This claim is immediate from the fact that $F_{n}(0)>0$ and $F_{n}(\varepsilon)$ is continuous.

To complete the proof of the lemma, take

$\varepsilon_{n} \doteq \min \left\{\frac{1}{n}, \varepsilon^{*}\right\}$

Now, by construction, it follows that the sequence $\left\langle\varepsilon_{n}\right\rangle_{n=1}^{\infty}$ converges to zero and

$F_{n}\left(\varepsilon_{n}\right)>0$

for all $n \geq N$. Consequently,

$\tilde{p}_{d(n)}\left(\jmath \omega, q, \varepsilon_{n}\right) \neq 0$

for all $\omega \in \mathbf{R}$ and $q \in Q_{\beta}$. This implies that

$\tilde{r}_{\max }\left(d(n), \varepsilon_{n}\right)>\beta$

for all $n \geq N$ The proof of the lemma is now complete.

\section{Acknowledgements}

This work was performed while B.R. Barmish was a Senior NATO Guest Fellow at CENS-CNR, Torino (Italy). The support of Professor A.R. Meo is gratefully acknowledged. Partial support of this project was also provided by the U.S. National Science Foundation under Grants ECS-8612948 and ECS-9096109, by the U.S. Air Force Office of Scientific Research under Grant AFOSR-88-0020 and by Honeywell and Boeing.

The authors are especially grateful to Dr. F. Kraus of ETH, Zurich. In his detailed comments on an earlier draft of this paper which appeared in the Proceedings of the 1989 CDC (see Barmish, Khargonekar, Shi and Tempo [13]), he exposed an error motivating this corrected version of the work.

\section{References}

[1] J C Doyle, Structured uncertainty in control system design, Proceedings of the IEEE Conference on Decision and Control, Ft Lauderdale, FL (1985) 
[2] A Packard, What's new with $\mu$ ? Ph.D Dissertation, Department of Mechancal Engineenng, University of Calıforna, Berkeley, CA (1987)

[3] V.L Khantonov, Asymptotic stability of an equilıbnum position of a family of systems of linear differential equations, Differential'nye Uraveniya 14 (1978) 1483-1485

[4] B.R. Barmish, New tools for robustness analysis, Proceedings of the IEEE Conference on Decision and Control, Austın, TX (1988).

[5] E I Jury, Robustness of discrete systems A review, to appear.

[6] A C. Bartlett, C V Hollot and L. Huang, Root locations of an entıre polytope of polynomials- It suffices to check the edges, Math Control Signals Systems 1 (1988) 61-71

[7] R R E. de Gaston and M.G Safonov, Exact calculation of the multiloop stability margin, IEEE Trans Automat Control 33 (1988) 156-171

[8] R.M Biernack,, H Hwang and S P Bhattacharryya, Robust stability with structured real parameter perturbatıons, IEEE Trans Automat Control 32 (1987) 495-506
[9] D Hinnchsen and A S Pntchard. New robustness results for linear systems under real perturbations, Proceedings of the IEEE Conference on Deciston and Control, Austın, TX (1988)

[10] B R Barmish, A generalızation of Khantonov's four polynomal concept for robust stability problems with linearly dependent coefficient perturbations, IEEE Trans Automat Control 34 (1989) 157-165

[11] J Ackermann, $\mathrm{HZ} \mathrm{Hu}$ and D. Kaesbauer, Robustness analysis A case study, Proceedings of the IEEE Conference on Decision and Control, Austın. TX (1988)

[12] C Berge, Topological Spaces (Oliver and Boyd, London, 1963)

[13] B R Barmısh, P P Khargonekar, Z.C. Shu and R. Tempo, A pitfall in some of the robust stability literature (prelimunary version of this paper), Proceedings of the IEEE Conference on Decision and Control, Tampa, FL (1989) 